\title{
The Intersection of Depressive Symptoms, Adverse Childhood Experiences, and Protective Factors Among Adolescents: Epidemiological Evidence from Minnesota, 2016 and 2019
}

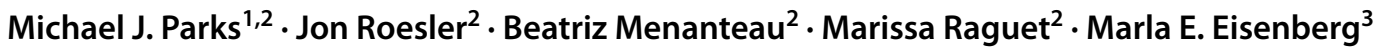

Accepted: 6 January 2022 / Published online: 26 January 2022

(c) The Author(s), under exclusive licence to Springer Nature Switzerland AG 2022

\begin{abstract}
Prevalence of mental health problems among US youth has increased in recent years, and there is a dearth of epidemiologi$\mathrm{cal}$ research at the state level that integrates risk and protective factors into population-based surveillance. We utilized the developmental assets framework to measure protective factors; we assessed (1) prevalence of depressive symptoms, high adverse childhood experiences (ACEs; $\geq 4$ ACEs), and few developmental assets ( $\leq 2$ assets) over time, and ( 2 ) associations among these three phenomena. Using 2016 and 2019 Minnesota Student Survey data, we utilized descriptive statistics, multivariable logistic regression, and post-estimation analyses (adjusting for school clustering and demographics). Using pooled data, we examined how high ACEs and few assets predicted depressive symptoms and we tested three-way interactions for high ACEs, few assets, and survey year. There were statistically significant increases in prevalence of depressive symptoms, high ACEs, and few assets between 2016 and 2019. High ACEs ( $\mathrm{AOR}=2.74 ; 95 \% \mathrm{CI}=2.61,2.89)$ and few assets $(\mathrm{AOR}=3.13 ; 95 \% \mathrm{CI}=3.00,3.26)$ were positively related to depressive symptoms; interactions were statistically significant. Additive interactions showed that, compared to their counterparts, adolescents with high ACEs and few assets had the highest prevalence of depressive symptoms, and this group exhibited the largest increase in prevalence between 2016 and 2019. Depressive symptoms are highest among adolescents with high risk and few protective factors, and recent increases in the prevalence of depressive symptoms appear to have disproportionately occurred among these adolescents. We offer a potential roadmap for following the Centers for Disease Control and Prevention recommendations to integrate ACEs and protective factors into local public health efforts.
\end{abstract}

Keywords Adverse childhood experiences $\cdot$ Youth mental health $\cdot$ Protective factors $\cdot$ Population-based prevention $\cdot$ Public health practice

\section{Introduction}

Mental health problems among young people have become pressing public health concerns. The prevalence of depression and depressive symptoms among US youth was relatively high but stable from 2006 to 2011 (Keyes

Michael J. Parks

park0614@umn.edu; michael.parks@ state.mn.us

1 Center for Applied Research and Educational Improvement, University of Minnesota, Minneapolis, MN, USA

2 Injury and Violence Prevention Section, Minnesota Department of Health, St. Paul, MN, USA

3 Department of Pediatrics, Medical School, University of Minnesota, Minneapolis, MN, USA et al., 2020; Mojtabai et al., 2016); depression then increased markedly since 2012, with dramatic increases occurring since approximately 2015 (Keyes et al., 2019, 2020). Increases in depressive symptoms since 2012 occurred across many age groups, but the prevalence of past-year major depressive episodes increased most rapidly among adolescents, with an increase from approximately $9 \%$ in 2012 to almost 13\% in 2016 (Lu, 2019; Weinberger et al., 2018). There are no definitive theories or hypotheses on why these increases are occurring. Some have suggested that overarching social change (e.g., delayed maturation among adolescents) has shifted adolescents' lives, increasing the likelihood of distress; however, recent increases in depressive symptoms outpace the timeframe within which social change at a societal level typically occurs (Keyes et al., 2019). Depressive symptoms are more endemic to 
US youth, as depressive symptoms have been increasing across all recent cohorts of youth from 2012 onward, and it is possible that a range of phenomena is linked to these increases, such as heavy use of smart phones and social media, increasing bullying, and/or declining hours of nightly sleep (Keyes et al., 2019).

Consequently, mental health problems among young people will be central public health concerns for the foreseeable future (Golberstein et al., 2019, 2020), and it is a priority to not only document trends but also predictors of depression (Ivey-Stephenson et al., 2020; Keyes et al., 2020). Documenting these two aspects of depression epidemiology is critical for state public health efforts focused on youth mental health, as it would provide up-to-date details on increasing trends in mental health problems as well as modifiable factors related to depression that could be addressed via programs and policies. There has been a steady amount of research related to depression prevalence at the national level but there have been limited analyses of state-level prevalence, which could be more fruitful for state public health efforts. Moreover, there is limited research on state-level trends in depressive symptoms that has simultaneously assessed trends in the prevalence of correlates and predictors of depression. The Centers for Disease Control and Prevention (CDC) has called for more systematic efforts to integrate correlates of youth mental health into local and state public health surveillance and programs, and namely the correlates of adverse childhood experiences (ACEs) and protective factors (Centers for Disease Control \& Prevention, 2019).

ACEs have garnered substantial attention, particularly as they pertain to depression and well-being among young people (Finkelhor, 2020). ACEs are measured as exposure to a set of potentially traumatic events in childhood (before age 18), including child abuse, parental substance abuse, parental incarceration, among others (Felitti et al., 2019). ACEs are linked to depressive symptoms (Bomysoad \& Francis, 2020; Choi et al., 2020; Crouch et al., 2020; Elmore \& Crouch, 2020), and they can be deleterious for physical and behavioral health (Dube et al., 2003; Felitti et al., 2019; Javier et al., 2019; Parks et al., 2018). The relationship between ACEs and depressive symptoms is even more stark for youth with cumulative ACEs (Turney, 2020), which is often measured as the presence of 4 or more ACEs (Duke et al., 2010; Forster et al., 2017). The prevalence of ACEs has remained relatively high and stable in recent years (Sacks \& Murphey, 2018; Sacks et al., 2014). It is important to note that while ACEs are associated with multiple negative health-related outcomes, both in the short and long term, there is no definitive set of ACEs (Finkelhor, 2020), and therefore, ACE measures can often miss other traumatic experiences such as neighborhood violence, discrimination, among others.
The Centers for Disease Control and Prevention (CDC) has called for more systematic efforts to integrate protective factors into local and state public health surveillance and health promotion strategies because protective factors can encourage positive outcomes despite the presence of risk (Centers for Disease Control \& Prevention, 2019). Protective factors span from individual characteristics to neighborhood conditions, and they can help youth who are at risk for depressive symptoms and ACEs (Elmore et al., 2020; Hostinar \& Miller, 2019; Liu et al., 2020). As noted by the CDC (Centers for Disease Control \& Prevention, 2019), if present in a young person's life, protective factors can reduce the likelihood of negative mental, physical, and environmental health outcomes (Fergus \& Zimmerman, 2005; Resnick et al., 1997). Protective factors have far-reaching implications, particularly in relation to both depressive symptoms and ACEs (Eiden et al., 2020; Fergus \& Zimmerman, 2005; Hostinar \& Miller, 2019; Resnick et al., 1997; Scales et al., 2004), and they are modifiable targets for programmatic and policy efforts geared toward healthy youth development (Catalano et al., 2012; Hostinar \& Miller, 2019; Masten \& Motti-Stefanidi, 2020; Patton et al., 2016; Syvertsen et al., 2019).

Even in the face of adversity, healthy youth development is possible with the assistance of protective factors that range from internal processes such as a sense of empowerment to external processes such as positive relationships with adults (Fergus \& Zimmerman, 2005; Scales et al., 2000). Resilience is the process of fostering positive outcomes despite risk (Henry et al., 2015; Masten, 2001; Wright et al., 2013), and protective factors increase the likelihood of resilience (Hays-Grudo \& Morris, 2020; Morris et al., 2021; Wright et al., 2013). Protective factors can contribute to the dynamic process, or the capacity of a dynamic system, that allows youth to adapt successfully to challenges or disturbances (e.g., ACEs) that threaten healthy development (Masten, 2015; Wright et al., 2013). Depression is one possible negative adaptation in presence of risk, and protective factors can foster better outcomes, such as the absences of depression (Henry et al., 2015; Wright et al., 2013).

Meso- to macro-level threats, such as the COVID-19 pandemic and its impact on the economy, have potential to influence risk factors such as ACEs as well as mental health problems among young people (Bryant et al., 2020; Golberstein et al., 2019, 2020). Therefore, protective factors and phenomena such as resilience, which have always been important, have become even more critical in recent years (Masten \& Motti-Stefanidi, 2020). Yet, despite the CDC's calls to track the prevalence of ACEs and protective factors among youth and their relation to mental health problems, surveillance by local and state public health agencies have not consistently tracked ACEs and key protective factors for depressive symptoms among youth. For local public health 
efforts to successfully foster resilience, promote protective factors, and reduce risk, it is vital to first conduct local assessments of issues such as prevalence of protective factors, risk factors such as ACEs, and negative adaptations to risk such as depression (Winslow et al., 2013), but there are no established roadmaps for such assessment in local public health. Local public health networks tend not to have a consistent set of frameworks and measures that can be used to assess the prevalence of ACEs and protective factors. For example, the CDC highlights a range of protective factors that are important for mental health outcomes (Centers for Disease Control \& Prevention, 2019), but it is often difficult to prioritize which protective factors to track without a clearly defined framework and set of measures.

\section{Current Study}

In this paper, we examine depressive symptoms among Minnesota youth. Minnesota has witnessed increases in the prevalence of mental health problems among adolescents (Minnesota Department of Health, 2019), and consequently, Minnesota serves as a good case study to assess how national trends are operating at the state level. For instance, $18 \%$ of students surveyed in 2016 reported long-term mental health, emotional, or behavioral problems, compared to $30 \%$ in 2019 (Minnesota Department of Health, 2019).

The field of youth mental health has provided previous studies on how exposure to multiple adversities in the absence of protective factors predicts worse mental health outcomes (e.g., Hays-Grudo \& Morris, 2020). Yet, there is a need for systematic approaches to integrating ACEs and protective factors into population-based public health promotion strategies in order to address mechanisms that influence mental health as well as a range of mental, physical, and environmental health outcomes for youth (Centers for Disease Control \& Prevention, 2019). There is a dearth of research that provides examples of data, frameworks, and measurements that addresses the need for integration of ACEs and protective factors into ongoing population-based public health efforts.

We address these gaps in two primary ways. First, we assess prevalence of depressive symptoms, ACEs, and protective factors among young people in Minnesota, and we examine how the prevalence of all three phenomena has changed from 2016 to 2019 , a period when the prevalence of depressive symptoms has increased at the national level. By using unique state-level data, the project provides information that could similarly be gathered across other states by local public health agencies. Second, we consider how ACEs and protective factors relate to depressive symptoms, and how these associations may vary over time, by utilizing measures that can be easily translated to local communities.
We contend that this project provides a roadmap for public health practitioners that can assist in measuring, documenting, and reporting measures in response to CDC recommendations.

For ACEs, we use a measure that captures the prevalence of youth who are most at risk for depressive symptoms by assessing cumulative ACEs (i.e., 4 or more ACEs). We utilize the developmental assets framework ([DAF]; Scales, 1999) to measure and assess protective factors. The conceptual link between DAF and positive or negative outcomes in the presence of risk is understudied. However, there are clear and strong overlaps between recent waves of resilience research and the DAF (Henry et al., 2015; Sesma et al., 2013). DAF was designed to identify predictors of positive outcomes, and consequently positive youth development (Sesma et al., 2013). These predictors of positive outcomes are the main point of overlap between research on resilience and the DAF, and they are the most beneficial aspect of the DAF for public health assessment and surveillance. The DAF argues that protective factors can be assessed as internal and external assets for youth (hereafter referred to as "assets" in accordance with the framework), and these assets can be measured in a dichotomous manner that captures prevalence estimates of low and/or high levels of assets among youth (Leffert et al., 1998; Scales, 1999). These assets overlap with the protective factors (Luthar et al., 2000), promotive factors (Sameroff et al., 1997; Wright et al., 2013), or other assets (Masten et al., 2009) articulated in resilience research, as all of these constructs positively predict healthy development despite the presence of risk (Sesma et al., 2013; Wright et al., 2013), such as in this study, the potential absence of depression despite the presence of high ACEs.

The DAF was also designed to provide a framework for creating easily accessible language and measures that can act as catalysts for community mobilization and action in the pursuit of positive youth outcomes, making it ideal for public health assessments and communication (Sesma et al., 2013). DAF from the Search Institute offers a clear set of measurements for protective factors in addition to a roadmap and an established track record for generating translatable prevalence estimates of protective factors. Research on protective factors often focuses on smaller sample sizes that include lengthy measures with strong psychometrics; however, dichotomous and prevalence measures, such as those offered by DAF, are easily translated to communities and usable in surveillance and public health practice (Briney et al., 2012; Leffert et al., 1998).

Another key point of overlap between resilience research and the DAF concerns cumulative protection, that is, the presence of multiple protective factors in an individual's life (Hays-Grudo \& Morris, 2020; Hays-Grudo et al., 2021; Sesma et al., 2013; Wright et al., 2013). Research on the DAF consistently shows that the power of assets lies in 
the cumulative pile-up of effects across multiple contexts (Sesma et al., 2013). This is linked to resilience research in that it is unlikely that a single asset is most important for positive adaptation or positive development since positive development can unfold through a range of pathways (Masten, 2015; Scales et al., 2004; Sesma et al., 2013). The DAF contends that multiple assets can be integrated into a single prevalence estimate that captures cumulative assets, or the number of assets present in an individual's life, helping local public health agencies consider a range of factors rather than attempting to prioritize a single asset to track. A growing area of research takes seminal developmental work (e.g., Cicchetti et al., 2000; Masten, 2001; Rutter, 1987; Sameroff, 1975; Werner \& Smith, 1992), and expands it by measuring and assessing the impact of cumulative protective factors among youth (Bethell et al., 2019; Hays-Grudo et al., 2021; Morris et al., 2021; Syvertsen et al., 2019). This research shows that cumulative protective factors can buffer against the deleterious effects of ACEs and promote healthy youth development (Hays-Grudo et al., 2021; Syvertsen et al., 2019; Morris et al., 2021; Bethell et al., 2019). Moreover, this research indicates that the interaction of ACEs and cumulative protective factors plays a pivotal role in adolescent health, as risk for poor health and developmental outcomes is highest for those who report high ACEs and few protective factors (Morris et al., 2021; Bethell et al., 2019). Consequently, the presence of ACEs increases risk for negative health outcomes such as depression, cumulative protection can encourage the process of positive outcomes despite risk, and risk is highest for those who experience adversity and low cumulative protection. The DAF offers one approach for assessing these relationships and processes, and it has a clear set of constructs and methods that can operate as a roadmap for local public health efforts.

\section{Research Questions}

This project addresses three main research questions. Research Question 1 (RQ1) asks: Did depressive symptoms, high ACE scores, and few assets increase in 2019 compared to 2016? We expect that the prevalence of depressive symptoms in Minnesota increased in 2019 compared to 2016, similar to national level trends, based on preliminary findings that mental health problems have increased among Minnesota adolescents (Minnesota Department of Health, 2019). Since ACEs and assets are related to depressive symptoms in previous research, it is possible that the prevalence estimates of high ACEs and few assets will have similarly changed in that same timeframe. However, it is possible that other factors related to depression among adolescents (e.g., racism, poverty) has increased over this time frame as well, and there is limited research on the prevalence of ACEs and assets at the state level; therefore, we did not make specific hypothesis about the prevalence of high ACEs and few assets.

Research Question 2 (RQ2) addresses the relationships among depressive symptoms, high ACEs, and few assets. Specifically, RQ2 asks: How do high ACEs and few assets independently and interactively predict depressive symptoms in 2016 and 2019 (using a pooled sample)? Based on research previously mentioned, we expect high ACEs and few assets to both independently predict depressive symptoms in both years and interactively relate to depressive symptoms-i.e., we expect direct effects for high ACEs and few assets, and that depressive symptoms will vary according to the combination of high ACEs and few assets (e.g., adolescents with high ACEs and few assets will be most at risk for depressive symptoms). Finally, in Research Question 3 (RQ3), we consider disparities in depressive symptoms according to high ACEs and few assets, focusing primarily on whether disparities have gotten more dramatic over time. That is, RQ3 asks: Does the change in depressive symptom prevalence in 2019 compared to 2016 differ according to high ACEs and few assets?

\section{Methods}

\section{Data and Sample}

Data came from the 2016 and 2019 Minnesota Student Survey (MSS), which is a statewide survey conducted every 3 years in Minnesota public schools among all students in grades $5,8,9$, and 11 . We excluded fifth grade students from the current analysis because they did not receive questions about ACEs. Approximately, $85 \%$ and $81 \%$ of public school districts in the state participated in 2016 and 2019, respectively. The MSS data are used as a census of all adolescents in Minnesota, and the data are the primary source for population-based surveillance of this age group in the state. The study sample consisted of 110,422 adolescents who provided data in 2016, and 105,014 who provided data in 2019. On average, adolescents were 14.8 years old $(\mathrm{SD}=1.32$, range $=12$ to 19$)$. The MSS sample is evenly distributed in terms of sex, and the distribution across grades $(8,10$, and 11) was even across 2016 and 2019 (see Table 1). Most adolescents in the MSS identified themselves as nonHispanic white in both years (69.2\% in 2016 and 2019). In terms of SES measures, most adolescents planned on attending a 4-year college (70.5\% in 2016, and 67.9\% in 2019), and $4.4 \%$ of adolescents skipped meals due to financial reasons in 2016, while $4.1 \%$ did so in 2019. (For more details on the demographic characteristics of the sample, see Measures and Table 1.) The Minnesota Department of Health's IRB approved the project and data collection. The study was 
Table 1 Demographic characteristics: Minnesota Student Survey 2016 and 2019

\begin{tabular}{llll}
\hline & 2016 & 2019 & \\
Demographic characteristics & $\%$ & $\%$ & $p$-value \\
\hline Grade & & & $<.001$ \\
8th & 36.14 & 36.72 & \\
9 th & 35.01 & 35.14 & \\
11th & 28.85 & 28.14 & \\
Sex & & & $<.001$ \\
Male & 49.25 & 48.02 & \\
Female & 50.75 & 51.98 & \\
Race/ethnicity & & & $<.001$ \\
Non-Hispanic White & 71.58 & 71.95 & \\
Non-Hispanic Black & 5.14 & 6.00 & \\
Hispanic & 9.03 & 5.60 & \\
Asian & 5.65 & 6.55 & \\
American Indian/Native American & 1.18 & 1.18 & \\
Multiple/another race/ethnicity & 7.43 & 8.72 & \\
Socioeconomic status & & & \\
College plans & & & $<.001$ \\
Yes & 70.52 & 67.85 & \\
No & 29.48 & 32.15 & \\
Skip meals & & & $<.01$ \\
Yes & 4.39 & 4.11 & \\
No & 95.61 & 95.89 & \\
Total $N$ & 110,422 & 104,530 & \\
\hline
\end{tabular}

$p$-values were based on chi-square tests; total $N=214,952$

performed in accordance with the 1964 Declaration of Helsinki and its later amendments.

\section{Measures}

\section{Depressive Symptoms}

We examined depressive symptoms via a 2-item screener (the Patient Health Questionnaire, PHQ-2). The PHQ-2 has been used as clinical screener for depression, and it is a reliable measure of depressive symptoms among adolescents (Kroenke et al., 2003; Spitzer et al., 1999). The two screener questions ask how often in the past 2 weeks participants (1) had "little interest or pleasure in doing things" and (2) were "feeling down, depressed or hopeless" (range: $0=$ not at all to $3=$ nearly every day). Following previous research (Richardson et al., 2010), we aggregated the 2 items (a total sum ranging from 0 to 6 ) and created a dichotomous measure, which was generated by using a threshold total score of greater than or equal to 3 ( $1=$ presence of depressive symptoms, $0=$ no depressive symptoms). This threshold is a reliable measure for DSM-IV criteria for depressive symptoms among adults and youth (Richardson et al., 2010).

\section{Developmental Assets}

Following past research (Leffert et al., 1998), we used a total of 10 assets available in the MSS that corresponded with the DAF. In spite of the limited number of assets available in the MSS, we included assets from all eight of the major categories of assets in the DAF: support; empowerment; boundaries and expectations; constructive use of time; commitment to learning; positive values; social competencies; and positive identity (Leffert et al., 1998; Scales, 1999). Each of these assets is described here, and the specific measures and their properties used in the analysis are described in Table 2. Support involves family support, positive family communication, receiving support from other adults that are nonparents, and support from neighbors or local communities (Scales, 1999). Empowerment entails adolescents having roles in family and communities, as well as a sense of safety (Leffert et al., 1998; Scales, 1999). Boundaries and expectations involve clear rules set by families and positive peer influence, including norms and expectations about substance use (Scales, 1999). Constructive use of time can capture time spent engaged in prosocial activities, such as the number of activities within which students are engaged (Leffert et al., 1998; Scales, 1999). Commitment to learning includes school bonding, school engagement, and achievement motivation (Scales, 1999). Positive values include a range of possible values, with beliefs about alcohol use as one indicator (Leffert et al., 1998). Social competency entails empathy, friendship skills, cultural competence, resistance skills, and conflict resolution (Scales, 1999). Positive identity involves self-esteem, sense of purpose, and positive views about the future (Scales, 1999).

These assets correspond with protective factors in resilience research (Sesma et al., 2013), and the DAF demonstrates two important aspects to consider for the measurement of assets. First, dichotomous and prevalence measures of assets are more easily translatable for use by communities and more easily usable in public health practice, as communities often respond well to knowing whether certain issues are common or less common (Briney et al., 2012; Leffert et al., 1998). Research on protective factors often focuses on smaller sample sizes that include measures with good psychometrics (e.g., scaled measures); however, dichotomous measures, as opposed to continuous measures, lend themselves better to applied purposes (Leffert et al., 1998). Consequently, we followed previous research (Leffert et al., 1998) and generated binary measures instead of continuous measure for a total of 10 assets. The cutoffs for each binary asset measure are listed in Table 2. All cutoffs for the measures were based on whether average scores were equal to 
Table 2 Developmental assets and their measurement

Asset category Asset used for category

Description of survey items

Reliability

coefficient

$(\alpha)$

Support

Support received from adults and community A 5-question scale with response options ranging

$\alpha=0.83$

from $1=$ not at all to $5=$ very much. The dichoto-

mous measure for support was based on an average

score of "quite a bit" or more-i.e., $>=4$ (Leffert

et al., 1998)

Empowerment

Included in family tasks

A 3-question scale that included items such as "I am included in family tasks and decisions" (range: $1=$ not at all or rarely to $5=$ extremely or almost always). The dichotomous measure of empowerment was based on an average score of "very or often"-i.e., > = 3 (Leffert et al., 1998)

Empowerment

Safety

Boundaries and expectations Parental norms

A 4-item scale that included items such as "I feel safe at home," and "I feel safe at school" (range: $1=$ strongly disagree to $4=$ strongly agree), with a cutoff for having the asset based on an average score of "agree" or more-i.e., $>=3$

Parental norms about substance use consisted of 4-item scale. It captured approval/disapproval of cigarettes, marijuana, alcohol, prescription drug use not prescribed to the student, and e-cigarette use (range: $1=$ not at all wrong to $4=$ very wrong). The dichotomous measure for anti-substance use norms among parents was based on an average score of "wrong" or "very wrong"-i.e., > = 3

Boundaries and expectations Peer norms

Same questions as parental norms were asked again but for peers rather than parents, and the dichotomous measure for anti-substance use norms among peers was similarly based on an average score of $>=3$

Constructive use of time Number of activities

Eight dichotomized activities (more than 1 day per): sports/rec/school/in-house teams; school-sponsored activities or clubs that are not sports such as drama; tutoring/homework help/academic program; leadership activities; artistic lessons; physical activities lessons not for sports teams; other community clubs such as 4-H; and religious activities such as a youth group. A dichotomy of greater than 2.5 activities versus less than 2.5 activities was used (Leffert et al., 1998)

Commitment to learning School engagement

A 7-item scale consisting of survey items such as, "being a student is one of the most important parts of who I am" (scale: 1 = strongly disagree to $4=$ strongly agree). The cutoff for having the asset was an average score of "agree" or more-i.e., > = 3

Positive values

Beliefs about alcohol use

A two-item scale that included beliefs about whether parents and other adults should clearly communicate with their children about the importance of not using alcohol, and whether drinking alcohol is never a good thing for anyone at their age (range: $1=$ strongly disagree to $5=$ strongly agree). The dichotomous metric for having this asset was based on an average score of "agree" or more-i.e., $>=4$ 
Table 2 (continued)

\begin{tabular}{|c|c|c|c|}
\hline Asset category & Asset used for category & Description of survey items & $\begin{array}{l}\text { Reliability } \\
\text { coefficient } \\
(\alpha)\end{array}$ \\
\hline Social competency & Empathy, emotional regulation, resistance & $\begin{array}{l}\text { A 9-item scale that included items such as "I express } \\
\text { feelings in proper ways," "I stay away from bad } \\
\text { influences," and "I am sensitive to the needs and } \\
\text { feelings of others" (range: } 1=\text { not at all or rarely to } \\
4=\text { extremely or almost always;); the dichotomous } \\
\text { cutoff for having this asset was an average score of } \\
\text { "very or often" or more-i.e., }>=3\end{array}$ & $\alpha=0.84$ \\
\hline Positive identity & Self-esteem, positive views about self & $\begin{array}{l}\text { A 5-item scale that included items such as "I feel } \\
\text { good about myself" (range: } 1=\text { not at all or rarely to } \\
4=\text { extremely or almost always;); the dichotomous } \\
\text { cutoff for having this asset was an average score of } \\
\text { "very or often" or more-i.e., }>=3\end{array}$ & $\alpha=0.84$ \\
\hline
\end{tabular}

or greater than "agree" for scales ranging from "strongly disagree" to "strongly agree," "quite a bit" for scales ranging from "not at all" to "very much," and "very or often" for scales ranging from "not at all or rarely" to "extremely or almost always" (see Leffert et al., 1998; Scales, 1999). Similar to past research (see Leffert et al., 1998), we found that this method of creating dichotomous measures loses variability, but in comparisons with standardized continuous distributions of the scales, there was good correspondence in the prevalence of adolescents who possessed the asset using the binary measures vis-à-vis adolescents who were 0.5 standard deviations above the mean using continuous distributions. This also increases the comparability of this study's findings to other research.

Second, these assets protect against negative outcomes despite risk, especially if individuals possess multiple assets. That is, the importance of cumulative assets for positive outcomes is a key tenet of the DAF. Research on the DAF indicates that youth or adolescents with few assets, measured as the bottom quartile of the distribution of cumulative assets, consistently experience negative health outcomes (Sesma et al., 2013). We measured assets in this manner: few assets versus not few assets. Consequently, we performed three steps to create our final measure that captured whether students had few development assets (Leffert et al., 1998; Scales, 1999): (1) we constructed binary measures of each asset by determining whether adolescents possessed a respective asset based on the distribution of an asset measure (i.e., distribution of continuous scales); (2) based on the 10 available development assets, we generated 10 dummy variables, and then created a count of the number of assets possessed by adolescents; and (3) after generating the count measure, we constructed a final dummy variable based on the distribution of the count metric (Scales, 1999). We generated a dummy variable that captured few developmental assets, which was defined as being in the bottom quartile of the distribution of the 10-asset count measure (Leffert et al.,
1998; Scales, 1999; Scales et al., 2000). This measure captured whether adolescents had few assets ( 2 or fewer assets) versus not few assets ( 3 or more assets). The final cumulative assets measure also had good psychometric properties $(\alpha=0.75)$. All factors contributed well to the overall measure based on item-test correlations and average inter-item covariance.

\section{ACEs}

Even though previous research on ACEs has used a number of ACEs that could amount to up to 10 total ACEs, not all ACEs from previous research were included in the MSS. Therefore, we followed previous research on ACEs that has used the MSS (Forster et al., 2017; Parks et al., 2018) and examined a total of seven separate ACEs that were available within the MSS data in 2016 and 2019: parental incarceration; parental alcohol abuse; parental illegal or prescription drug abuse; verbal abuse; physical abuse; exposure to parental intimate partner violence; and sexual abuse by a family member. We generated seven dummy variables $(1=$ yes, $0=$ no) for each ACE. Based on past research (Anda et al., 1999; Duke et al., 2010; Parks et al., 2018), we created a measure for high cumulative ACEs $(1=4$ or more ACEs, $0=3$ or fewer ACEs).

\section{Covariates}

Covariates included grade in school (grades 8, 9, and 11), sex, race/ethnicity, and socioeconomic status. Dummy variables were used for grades 8,9 , and 11. Sex was measured as $1=$ male, $0=$ female. Dummy variables were used for race/ethnicity (non-Hispanic white, non-Hispanic Black, Hispanic, Asian, American Indian/Native American, and another race/ethnicity option or multiple races/ethnicities). Race and ethnicity were included as a proxy for discrimination and racism. Socioeconomic status was assessed using 
(1) dummy variables for skipped meals due to financial reasons and (2) definitive plans to attend a 4-year college (see Parks et al., 2018; Patrick et al., 2020). Survey data from national surveys demonstrate that having definitive college plans (versus not having definitive plans to attend a 4-year college) can serve as a proxy for present and future SES (for example, see Patrick et al., 2020). Previous analyses have demonstrated that there are disparities in depression, high ACEs, and few assets, particularly according to SES. Consequently, we account for these disparities by adjusting for demographics, and we adjust for grade in school to account for any potential age effects.

\section{Statistical Analysis}

Addressing RQ1, we used descriptive statistics ( $\chi 2$ tests) to examine how the prevalence of depressive symptoms, high ACEs ( $>=4$ ACEs), and few assets ( $<=2$ assets) changed across survey years (2016 and 2019). For RQ1, we also compared demographics across 2016 and 2019 using descriptive analyses. We ran a series of multivariable logistic regression models to determine if the change between 2016 and 2019 in depressive symptoms, high ACEs, and few assets was statistically significant adjusting for age (grade in school), sex, race/ethnicity, SES, and school clustering (using a pooled sample). Logistic regression models are optimal because they allow for regression analyses of a binary outcome, and we can assess relationships (e.g., differences between survey years) adjusting for covariates. We use odds ratios, as they provide a metric for deciphering the strength of relationships/effect sizes. We used post-estimation (margins in Stata v.15) commands to obtain predicted probabilities for depressive symptoms, high ACEs, and few assets across survey years, adjusting for all other covariates and school clustering. We also conducted supplemental analyses for RQ1 that examined the differences in predicted probabilities between 2016 and 2019 for all demographic groups to ensure any differences were not the result of major differences across demographic groups-i.e., age effects. Students were nested within schools, and all analyses adjusted for this clustering by using cluster-adjusted standard errors.

We addressed RQ2 and RQ3 by using logistic regression models that used depressive symptoms as an outcome and high ACEs and few assets as primary predictors, adjusting for all covariates in previous models and school clustering. For RQ3, we tested interaction effects on multiplicative and additive scales (Dow et al., 2019). Our models had sufficient power to detect strong relationships for interactions in logistic regression models using log-odds and odds ratios (Demidenko, 2008). For additive scales, we predicted marginal probabilities with covariates at their mean values (Dow et al., 2019). For both interactions, we examined a three-way interaction between waves (2016 vs. 2019), high
ACEs ( $>=4$ ACEs vs. $<4$ ACEs), and few assets $(<=2$ assets vs. $>2$ assets). We provided post-estimation results for predicted scores of depressive symptoms across years for different groups based on possible combinations of high ACEs and few assets that result from the interaction (i.e., few assets $[<=2]$ and high ACEs $[>=4]$; few assets $[<=2]$ and not high ACEs $[<4]$; not few assets [ $>2]$ and high ACEs $[>=4]$; and not few assets $[>2]$ and not high ACEs $[<4]$ ). These predicted scores compared differences within survey years as well as differences in the change across survey years for the four possible combinations of high ACEs and few assets. All post-estimation comparisons adjusted for multiple comparisons using Bonferroni method. List-wise deletion was used for missing data, and supplemental analyses used multiple imputation for regression analyses and found no major changes in results and conclusions.

We also conducted supplemental analyses that used a higher cutoff for depressive symptoms since there is a tradeoff between sensitivity and specificity with different cutoffs for the PHQ-2 (Kroenke et al., 2003). The cutoff of greater than or equal to 3 is the optimal balance of sensitivity and specificity (Kroenke et al., 2003). However, a score of $>=4$ offers a slightly better specificity score and positive predictive value for clinical depression (but with a markedly lower sensitivity score; Kroenke et al., 2003). Consequently, we conducted supplemental analyses that used this $>=4$ cutoff to assess depressive symptoms using the measure with slightly better specificity.

\section{Results}

\section{Depressive Symptoms, High ACEs, and Few Assets Across Waves}

There were moderate and statistically significant increases in the adjusted prevalence of depressive symptoms, high ACEs, and few assets between 2016 and 2019 (see Fig. 1). Estimates for adjusted change across 2016 and 2019 for the entire population and each demographic group are presented in Supplemental Table 1. The pattern of change between 2016 and 2019 generally holds for percent change and absolute change across all demographic groups (Supplemental Table 1). The percent change was highest for prevalence of high ACEs, followed by prevalence of few assets and depressive symptoms (Supplemental Table 1). Due to the large sample size and small differences in raw change in prevalence, odds ratios were used to examine effect sizes for the change between 2016 and 2019, and to compare the effect size across the outcomes. For depressive symptoms, the odds ratio was $1.03(95 \% \mathrm{CI}=1.00,1.05)$, and the odds ratios were $1.21(95 \% \mathrm{CI}=1.15,1.28)$ for ACEs and $1.20(95 \%$ $\mathrm{CI}=1.14,1.25)$ for few assets. In 2019 relative to 2016, the 


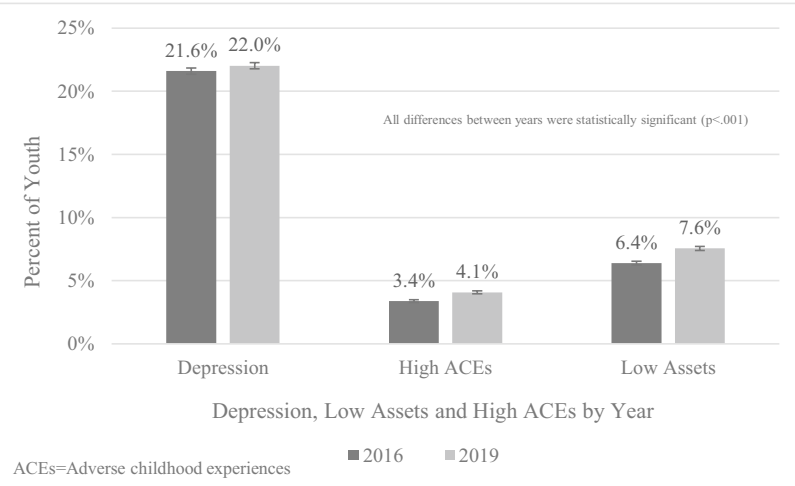

Fig. 1 Prevalence of depressive symptoms, high ACEs, and few assets by year among Minnesota youth

odds of experiencing depressive symptoms increased by $3 \%$, and the odds increased by $21 \%$ and $20 \%$ for experiencing high ACEs and few assets, respectively. These effect sizes indicate low to moderate increases between 2016 and 2019, with larger increases occurring for high ACEs and few assets compared to depressive symptoms.

\section{Predicting Depressive Symptoms with High ACEs and Few Assets}

Shown in Table 3 (Model 1), in the pooled sample, the odds of reporting depressive symptoms increased by $174 \%$ for adolescents who reported high ACEs compared to few/no ACEs, adjusting for covariates $(\mathrm{AOR}=2.74 ; 95 \%$ $\mathrm{CI}=2.61,2.89$ ). The odds of exhibiting depressive symptoms increased by $213 \%$ for adolescents who reported few assets ( 2 or fewer) relative to not few assets ( $\mathrm{AOR}=3.13$; $95 \% \mathrm{CI}=3.00,3.26$ ). The increase in depressive symptoms between 2016 and 2019 became nonsignificant after adjusting for high ACEs and few assets. Associations for covariates are shown in Table 3 (Model 1).

\section{Interaction Results}

For the three-way interaction term for survey year, high ACEs, and few assets, the joint test was statistically significant $(\chi 2=3000.42, \mathrm{df}=7, p<0.001)$. The additive threeway interaction was also statistically significant $(\chi 2=19.38$, $\mathrm{df}=4, p<0.001)$. Figure 2 and Table 4 demonstrate the key components of this three-way interaction via post-estimation predicted probabilities of depressive symptoms. Figure 2 shows the predicted probabilities of depressive symptoms for the four possible combinations of ACEs and assets. The high ACEs and few assets group (group 1) had the highest probability of depressive symptoms in both 2016 and 2019 , and this group exhibited the largest increase in percentage points between 2016 and 2019 (5.6\%; S.E. $=2.07$;
Table 3 Multiple logistic regression predicting depressive symptoms based on year, adverse childhood experiences, few assets, and demographic characteristics

\begin{tabular}{llll}
\hline & \multicolumn{3}{l}{ Model 1} \\
\cline { 2 - 4 } Variables & AOR & $(95 \%$ CI $)$ & \\
\hline Wave (vs. Year 2016) & & & \\
$\quad$ Year 2019 & 0.98 & $(0.96,1.01)$ & \\
High ACEs (4 or more) & 2.74 & $(2.61,2.89)$ & $* * *$ \\
Few assets & 3.13 & $(3.00,3.26)$ & $* * *$ \\
Grade (vs. 8th) & & & \\
$\quad$ 9th & 1.07 & $(1.03,1.11)$ & $* * *$ \\
$\quad$ 11th & 1.13 & $(1.08,1.18)$ & $* * *$ \\
Male & 0.51 & $(0.49,0.52)$ & $* * *$ \\
Race/ethnicity & & & \\
$\quad$ Non-Hispanic White (reference) & & & \\
$\quad$ Non-Hispanic Black & 1.17 & $(1.10,1.23)$ & $* * *$ \\
$\quad$ Hispanic & 1.22 & $(1.16,1.27)$ & $* * *$ \\
$\quad$ Asian & 1.29 & $(1.22,1.36)$ & $* * *$ \\
$\quad$ American Indian/Native American & 1.38 & $(1.25,1.52)$ & $* * *$ \\
$\quad$ Multiple/another race/ethnicity & 1.46 & $(1.40,1.52)$ & $* * * *$ \\
College plans & 0.68 & $(0.66,0.70)$ & $* * * *$ \\
Skips meals & 2.34 & $(2.23,2.46)$ & $* * *$ \\
\hline
\end{tabular}

Note. $N=214,952$; Model 1 does not include interaction terms ${ }^{* * * *} p<0.001$

$p<0.05)$. The 2 groups with few assets but not high ACEs (group 2) and not few assets but high ACEs (group 3) had relatively similar predicted scores for depressive symptoms, and both groups had higher percentages than the group with not few assets and not high ACEs (group 4). The two groups of either few assets or high ACEs (groups 2 and 3) also exhibited increases in depressive symptoms between 2016 and 2019 (respectively, change between years was 1.9\% and $0.7 \%$ ); however, the increases for both groups were not statistically significant. The group with not few assets and not high ACEs (group 4) had the lowest predicted score for depressive symptoms in both 2016 and 2019, and this group exhibited a small, nonsignificant decrease in depressive symptoms between 2016 and $2019(-0.5 \%$; S.E. $=0.20$; $p>0.05$ ).

Table 4 presents differences in depressive symptoms within and across years for all groups based on the four possible combinations of assets and ACEs. All group differences within survey years were statistically significant, except for the difference between the two groups that had either high ACEs without few assets (group 3) or few assets without high ACEs (group 2). The difference in the change in depressive symptoms between 2016 and 2019 was larger and statistically significant for the group with few assets and high ACEs (group 1) compared to the group without few assets and without high ACEs (group 4; difference $=6.16 \%$; 
Fig. 2 Adjusted probabilities of reporting depressive symptoms by assets and ACEs groups, 2016 and 2019
Table 4 Adjusted predicted probabilities for depressive symptoms across years and by ACEs and assets

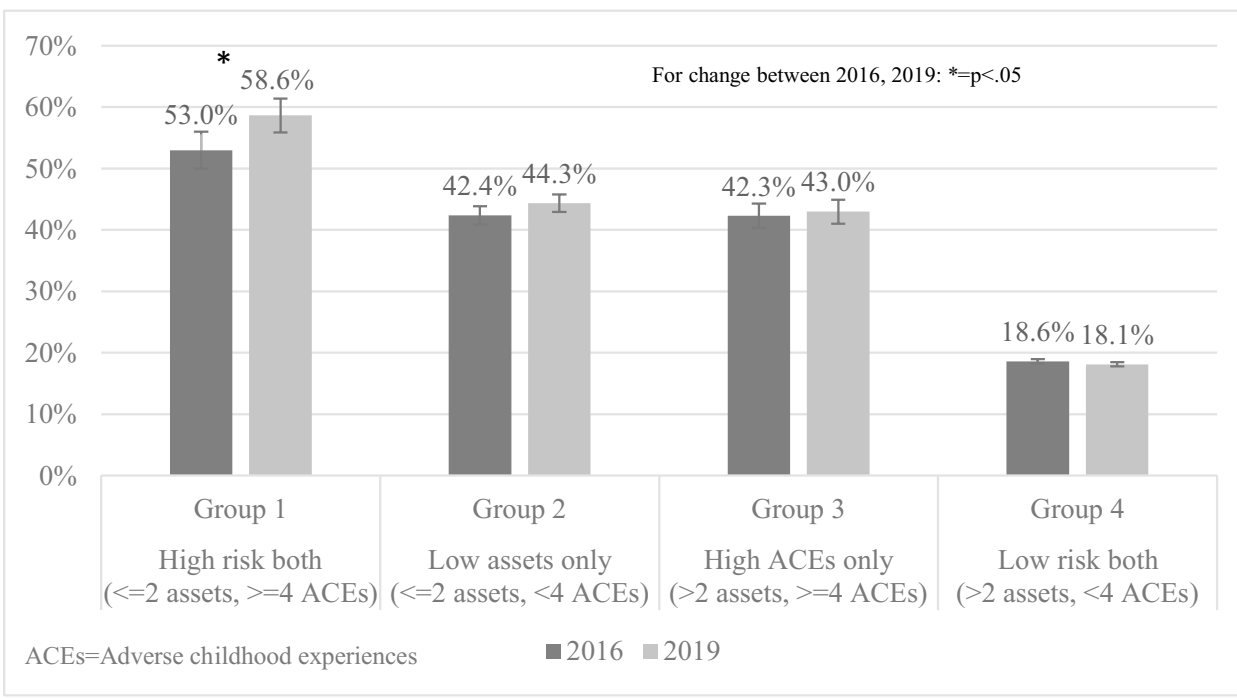

\begin{tabular}{|c|c|c|c|c|c|}
\hline \multirow{3}{*}{$\begin{array}{l}\text { Differences within and } \\
\text { across years by groups } \\
\text { Group } 1 \text { vs. group } 2\end{array}$} & \multirow{2}{*}{\multicolumn{2}{|c|}{$\begin{array}{l}\text { Differences within } 2016 \\
\% \text { (S.E.) }\end{array}$}} & \multirow{2}{*}{\multicolumn{2}{|c|}{$\begin{array}{l}\text { Differences within } 2019 \\
\% \text { (S.E.) }\end{array}$}} & \multirow{2}{*}{$\begin{array}{l}\begin{array}{l}\text { Differences in } \\
\text { between-year change }\end{array} \\
\% \text { (S.E.) }\end{array}$} \\
\hline & & & & & \\
\hline & $10.61(1.72)$ & $* * *$ & $14.30(1.54)$ & $* * *$ & $3.69(2.33)$ \\
\hline Group 1 vs. group 3 & $10.67(1.87)$ & $* * *$ & $15.70(1.72)$ & $* * *$ & $5.03(2.58)$ \\
\hline Group 1 vs. group 4 & 34.35 (1.54) & $* * *$ & $40.51(1.39)$ & $* * *$ & $6.16(2.07)$ \\
\hline Group 2 vs. group 3 & $0.07(1.24)$ & & $1.40(1.20)$ & & $1.33(1.71)$ \\
\hline Group 2 vs. group 4 & $23.74(0.76)$ & $* * *$ & $26.21(0.73)$ & $* * *$ & $2.47(1.03)$ \\
\hline Group 3 vs. group 4 & $23.67(1.02)$ & $* * *$ & $24.81(1.00)$ & $* * *$ & $1.14(1.03)$ \\
\hline
\end{tabular}

${ }^{* * *}=p<.001 ; * *=p<.01 ; *=p<.05 ;+=p<.10$

Comparisons were adjusted for multiple comparisons using Bonferroni correction

Estimates adjusted for all covariates and for school clustering

ACEs, adverse childhood experiences

Group description:

Group 1 -high risk for both ( $<=2$ assets, $>=4$ ACEs)

Group 2-few assets only ( $<=2$ assets, $<4$ ACEs)

Group 3-high ACEs only ( $>2$ assets, $>=4$ ACEs)

Group 4 -low risk for both ( $>2$ assets, $<4$ ACEs)
S.E. $=2.07 ; p<0.05)$. Put differently, the change between 2016 and 2019 was most dramatic for this group of adolescents with few assets and high ACEs relative to adolescents with relatively low risk (not few assets and not high ACEs). Further, the disparity in depressive symptoms experienced by adolescents with few assets and high ACEs worsened over time relative to adolescents who did not have few assets and high ACEs.

\section{Supplemental Results}

All results described above held using the depressive symptom measure with greater specificity $(>=4)$. The percentage point change and percent change were larger for the measure with higher specificity. For instance, the odds ratio was $1.11(95 \% \mathrm{CI}=1.07,1.14)$, indicating that in 2019 relative to 2016 , the odds of experiencing depressive symptoms increased by $11 \%$, which is a larger effect size compared to the measure depression measure of $>=3$. In addition, unlike the $>=3$ measure, the adjusted absolute change between 2016 and 2019 was statistically significant and the change was similar and statistically significant across all demographic groups. Moreover, with the $>=4$ measure, the increase in depressive symptoms between 2016 and 2019 remained statistically significant after adjusting for high ACEs and few assets $(\mathrm{AOR}=1.07 ; 95 \% \mathrm{CI}=1.04,1.10)$. 


\section{Discussion}

Prevalence of depressive symptoms, high ACEs, and few assets is a challenge in Minnesota. In 2019, approximately $22 \%$ of adolescents exhibited depressive symptoms in Minnesota. We found that nearly $8 \%$ and $4 \%$ of Minnesota adolescents in 2019 reported few developmental assets and high ACEs, respectively. These results indicate that thousands of students are experiencing depressive symptoms, as well as low numbers of protective factors or high levels of risk. Additionally, in addressing RQ1, we found that the prevalence of depressive symptoms, high ACEs, and few developmental assets among adolescents in Minnesota have similarly increased since 2016. Put differently, the prevalence of high ACEs and few assets have increased in parallel fashion with depressive symptoms among Minnesota adolescents. However, the increase in depressive symptoms was dramatically smaller than the increase in prevalence of high ACEs and few assets. These results for RQ1 indicate that not only is depression increasing among adolescents, but the phenomena that increase risk for the negative outcome of depression have also increased, and have done so in a more pronounced fashion.

Research on mental health, resilience, and healthy youth development show that depression can be understood as a maladaptation or negative outcome in the presence of risk (Hays-Grudo \& Morris, 2020; Henry et al., 2015; Sesma et al., 2013; Wright et al., 2013), and ACEs increase the risk for negative health outcomes such as depression (Hays-Grudo \& Morris, 2020; Hays-Grudo et al., 2021; Wright et al., 2013). Moreover, protective factors can encourage resilience, or the occurrence of positive outcomes (i.e., no depression) despite the presence of risk (Henry et al., 2015; Masten et al., 2009; Wright et al., 2013). The DAF corresponds with this research in child and adolescent resilience, primarily through the constructs of protective factors as well as the notion that cumulative protection in the form of the number of protective factors in an individual's life provides the greatest chance for positive outcomes to occur, despite the presence of risk (Sesma et al., 2013). As expected, high ACEs and few assets independently predicted depressive symptoms in pooled analyses, and the inclusion of these measures as covariates made the difference between survey years nonsignificant, indicating the importance of both ACEs and assets for the change in depressive symptoms. Consequently, our results for RQ2 support previous research by demonstrating that (1) the presence of high ACEs increases risk for negative outcomes such as depression, (2) low protection in the form of few assets increases risk for negative outcomes, and (3) the interaction of assets and ACEs matters for risk, in that having more assets can protect against the risk of negative outcomes despite the presence of risk. Put differently, adolescents with either high ACEs or few assets were more at risk for depressive symptoms compared to adolescents without high ACEs or without few assets, and adolescents with high ACEs and few assets were at even more risk for depressive symptoms relative to all other adolescents, as these adolescents had low protection in the presence of high risk.

RQ3 focused on the intersection of cumulative protective factors and risk in the form of ACEs, particularly in association with recent increases in the prevalence of depression. We found that high ACEs and low assets interacted with survey year (2016 vs. 2019) in terms of predicting depression. We focus this discussion of RQ3 by considering the additive interaction results and specifically the prevalence of depressive symptoms across adolescents with high ACEs and/or few assets to highlight interaction results. Previous research has not considered how increases in prevalence of depressive symptoms over time vary across groups of adolescents with high ACEs and/or few assets. We were able to determine that adolescents with high risk (high ACEs) and low protection (few assets) were more at risk for depressive symptoms compared to their counterparts, and they disproportionately experienced the increases in prevalence of depressive symptoms in 2019 relative to 2016. That is, the prevalence of depressive symptoms increased most dramatically among adolescents with high ACEs and few assets relative to other groups of adolescents. The prevalence of depressive symptoms among adolescents without high ACEs and without few assets did not change between 2016 and 2019 (i.e., the most prevalent group of adolescents), indicating depressive symptoms prevalence is increasing primarily among adolescents with high risk for negative outcomes and few protective factors that buffer against this risk. The magnitude of the difference across survey years for adolescents without high ACEs and without few assets was not necessarily meaningful, whereas the increase among adolescents with high ACEs and with few assets was marked. It is also important to note that there were increases in the prevalence of depression among adolescents with high risk only (high ACEs/not few assets) or low protection only (not high ACEs/few assets), but these increases were also not nearly as marked.

As previously noted, there are no definitive theories or hypotheses on why increases in depressive symptoms, and based on this study, ACEs and few assets as well, are occurring among all youth. It is possible that overarching social change has shifted adolescents' lives, thus increasing the likelihood of distress (Keyes et al., 2019). It is also possible that these trends are endemic to US youth or that these patterns are limited to certain cohorts based on cohort-specific phenomena such as the Great Recession (Keyes et al., 2019). Similar to past research, we found that increased depressive 
symptoms, high ACEs scores, and few assets occurred across all age groups, which raises the possibility of period effects. Possible period effects could range from the influence of heavy smart phone and social media use to increased cyber bullying (Keyes et al., 2019).

\section{Implications for Local and State Public Health}

The CDC has called for systematic efforts to integrate ACEs and protective factors into state and local public health surveillance, particularly in relation to key public health issues such as youth mental health problems (Centers for Disease Control \& Prevention, 2019). However, there is limited research on how to systematically integrate consistent measures of ACEs and protective factors into local and statewide population-based surveillance. This project offers a roadmap for addressing these calls, relying on the DAF to measure and assess protective factors for positive youth outcomes.

All three measures (depressive symptoms, high ACEs, few assets) used in the current project were rooted in approaches that ensured high sensitivity in terms of accurately measuring the intended phenomena. Metrics for depressive symptoms and ACEs have been frequently used in research. In comparison, the current project used a metric for few assets that, while sound and based in past research, was recently introduced in epidemiological research (e.g., Hays-Grudo et al., 2021; Morris et al., 2021; Bethell et al., 2019). Prior studies on communitybased prevention indicates that the bottom quartile of the number of reported protective factors (i.e., $<2$ assets out of 10 in the current study) captures youth who definitively have low levels of protective factors (Leffert et al., 1998; Scales, 1999). The approach used here (i.e., a dichotomous measure that captures the prevalence of few assets) is different relative to measure rooted in continuous scales. Previous research has labeled these two approaches as "empirical" for continuous scales and "applied" for prevalence measures, primarily because the applied approach that uses dichotomous measure is more easily translated to communities and usable in public health practice (Briney et al., 2012; Leffert et al., 1998). The detailed information on the prevalence of few assets can be reported to local communities as an additional resource to guide local efforts focused on reducing risk and promoting protective factors. Further, the use of prevalence estimates can bypass the difficulties in reporting and explaining levels of continuous scales for protective factors relative to the ease of reporting prevalence estimates (Briney et al., 2012; Scales, 1999). The current study's approach, rooted in the DAF, offers local and state public health agencies a single measure to track over time since the DAF contends that cumulative protection is key for promoting positive youth outcomes, similar to how cumulative protective factors offers opportunity for resilience despite the presence of risk. Therefore, this approach might also help for surveillance purposes because a single measure of protective factors can be tracked and shared with communities and funding agencies.

In sum, in support of similar calls to action (Auerbach \& Miller, 2018; Hawkins et al., 2012; Hays-Grudo et al., 2021), our findings offer the key implication that state public health agencies should emphasize strategies that focus on tracking and promoting cumulative protective factors among youth. Public health efforts need to focus on maintaining and establishing capacity (internally in public health departments and state public health infrastructure, as well as externally in local communities) to document and disseminate prevalence of multiple protective factors. This capacity would help to guide evidence-based policies and interventions geared toward reducing risk and promoting protective factors for youth well-being, having implications for mental health and other important youth health outcomes such as violence, sexual violence, and substance use.

Additionally, our findings support arguments for universal programs and policies that holistically advance up-stream strategies that could prevent ACEs as well as increase cumulative protective factors, thereby supporting prevention across many areas. Evidence-based youth programs can increase protective factors at multiple levels, including peers, families, and communities (Oesterle et al., 2018). Such programs can also directly address ACEs. Public health researchers and practitioners need to address both the societal conditions that contribute to ACEs, as well as implement evidence-based programs that contribute to promoting cumulative protective factors that help all youth, especially youth exposed to ACEs. A prime example of such universal programs is the Communities That Care (CTC) model (Hawkins et al., 2012). CTC is rooted in principles from the field of prevention science, such as the social development model, with a focus on individual, community, family, and peer-group factors related to social and health problems. While we argue the measure of cumulative protective factors is helpful for surveillance purposes, we do not want to eliminate the importance of assessing the prevalence of separate protective factors within states and local areas-such assessments could help to determine if capacity is needed for addressing specific factors, which would allow for a tailoring to local areas. Public health agencies need to continue to implement issue-specific programs and policies in order to provide and improve access to treatment for depression among youth. Yet for population-based surveillance and reporting purposes, the metric used in the current project would provide a succinct and applied measure allowing state and local agencies to incorporate protective factors into ongoing efforts in accordance with CDC recommendations. 


\section{Limitations}

This study had several limitations. As with all secondary data analyses, this study is limited by the measures that were available in this surveillance instrument. The asset of boundaries and expectations is a measure that could be highly dependent on cultural context, and therefore, this measure may not translate across cultures and is a potential limitation of the current study. It is also important to note that the PHQ-2 is a limited measure of depression and it is primarily used to screen youth for depressive symptoms in order to provide a referral to a longer screening process as well as potential treatment in clinical settings (Kroenke et al., 2003, 2009). As noted, there are also tradeoffs in the different cutoffs for the PHQ-2; and to avoid any potential biases in the measure, we conducted supplemental analyses that used a higher cutoff for depressive symptoms that has a greater predictive value of clinical depression (Kroenke et al., 2003, 2009; Spitzer et al., 1999). Our results held in these supplemental analyses, and generally, the main patterns were even more pronounced with this PHQ-2 score that exhibited greater specificity.

Findings on the differences in depressive symptoms, ACEs, and assets between 2016 and 2019 could be the result of age, period, or cohort effects. The current study demonstrated that all trends in depressive symptoms, ACEs, and assets were present for all age groups, thus eliminating potential for age effects. However, we could not eliminate the possibility of period or cohort effects. It could be possible that overarching environmental issues have produced period or cohort effects, such as overall decreases in certain supports for youth over time (e.g., school programs) or trends in unmeasured stressors (e.g., discrimination, political climate). Research should examine additional years of data in the future and continue to examine trends in mental health problems, ACEs, and assets over time, specifically focusing on how age, period, or cohort effects might explain the patterns of increasing depressive symptoms, high ACEs, and few assets. Future surveillance efforts could also attempt to capture prevalence of few assets and high ACEs over time and across geographical space (e.g., counties, neighborhoods, schools). It is possible that local changes and phenomena have contributed to the trends found at the state level in the current study; for instance, changes in more local environments such as neighborhoods (e.g., racial/ ethnic segregation, changes in the concentration of poverty and wealth) could have contributed to aggregate trends at the state level in depressive symptoms, ACEs, and assets over time.

\section{Conclusion}

We found that the prevalence of depressive symptoms remains high in Minnesota and that this prevalence has increased in 2019 relative to 2016. Adolescents with high
ACEs and/or few assets were more at risk for depressive symptoms compared to their counterparts, as adolescents with high ACEs and/or few assets had the highest prevalence of depressive symptoms in general. Additionally, the increase in depressive symptoms prevalence in 2019 relative to 2016 was disproportionately experienced by adolescents with high ACEs and few assets. Public health agencies should develop capacity to engage in state and local level surveillance of mental health problems, ACEs, and protective factors among adolescents. Documenting the prevalence of high ACES and few assets appears to be a particular fruitful approach for local public health efforts. It may also prove useful to other public health efforts directed towards youth health promotion, many of which would benefit from an integration of risk and protective factors.

Supplementary Information The online version contains supplementary material available at https://doi.org/10.1007/s42844-022-00052-2.

Acknowledgements The authors would like to thank the MSS work group at the University of Minnesota for helpful feedback on this paper.

Author Contribution We have listed everyone who contributed significantly to the work.

Funding This publication was supported by the State of Minnesota as well as the Centers for Disease Control and Prevention [award numbers NU17CE924836 \& NUF2CE002476]. Data were collected with support from the State of Minnesota. The content is solely the responsibility of the authors and does not necessarily represent the official views of the Centers for Disease Control and Prevention.

\section{Declarations}

Ethics Approval The Minnesota Department of Health's IRB approved the project and data collection. The study was performed in accordance with the 1964 Declaration of Helsinki and its later amendments.

Consent to Participate All participants provided assent and parental consent to participate.

Conflict of Interest The authors declare no competing interests.

\section{References}

Anda, R. F., Croft, J. B., Felitti, V. J., Nordenberg, D., Giles, W. W. H., Williamson, D. F., \& Giovino, G. A. (1999). Adverse childhood experiences and smoking during adolescence and adulthood. Journal of the American Academy of Child \& Adolescent Psychiatry, 282(17), 1652-1658. https://doi.org/10.1001/jama.282. 17.1652 .

Auerbach, J., \& Miller, B. F. (2018). Deaths of Despair and building a national resilience strategy. Journal of Public Health Management and Practice, 24(4), 297-300. https://doi.org/10.1097/PHH. 0000000000000835.

Bethell, C., Jones, J., Gombojav, N., Linkenbach, J., \& Sege, R. (2019). Positive childhood experiences and adult mental and 
relational health in a statewide sample: Associations across adverse childhood experiences levels. JAMA Pediatrics, 173(11), e193007-e193007. https://doi.org/10.1001/jamapediatrics.2019. 3007.

Bomysoad, R. N., \& Francis, L. A. (2020). Adverse childhood experiences and mental health conditions among adolescents. Journal of Adolescent Health, 67(6), 868-870. https://doi.org/10.1016/j. jadohealth.2020.04.013.

Briney, J. S., Brown, E. C., Hawkins, J. D., \& Arthur, M. W. (2012). Predictive validity of established cut points for risk and protective factor scales from the communities that care youth survey. Journal of Primary Prevention, 33(5-6), 249-258. https://doi.org/10.1007/ s10935-012-0280-1.

Bryant, D. J., Oo, M., \& Damian, A. J. (2020). The rise of adverse childhood experiences during the COVID-19 pandemic. Psychological Trauma: Theory, Research, Practice, and Policy, 12, S193-S194. https://doi.org/10.1037/tra0000711.

Catalano, R. F., Fagan, A. A., Gavin, L. E., Greenberg, M. T., Irwin, C. E., Ross, D. A., \& Shek, D. T. L. (2012). Worldwide application of prevention science in adolescent health. The Lancet, 379(9826), 1653-1664. https://doi.org/10.1016/s0140-6736(12)60238-4.

Centers for Disease Control and Prevention. (2019). Preventing Adverse Childhood Experiences: Leveraging the Best Available Evidence. Atlanta, GA: National Center for Injury Prevention and Control, Centers for Disease Control and Prevention.

Choi, C., Mersky, J. P., Janczewski, C. E., Plummer Lee, C. T., Davies, W. H., \& Lang, A. C. (2020). Validity of an expanded assessment of adverse childhood experiences: A replication study. Children and Youth Services Review, 117, 105216. https://doi.org/10. 1016/j.childyouth.2020.105216.

Cicchetti, D., Toth, S. L., \& Maughan, A. (2000). An ecological-transactional model of child maltreatment. In Handbook of Developmental Psychopathology. https://doi.org/10.1007/978-1-46154163-9_37.

Crouch, E., Jones, J., Strompolis, M., \& Merrick, M. (2020). Examining the association between ACEs, childhood poverty and neglect, and physical and mental health: Data from two state samples. Children and Youth Services Review, 116, 105155. https://doi. org/10.1016/j.childyouth.2020.105155.

Demidenko, E. (2008). Sample size and optimal design for logistic regression with binary interaction. Statistics in Medicine, 27(1), 36-46. https://doi.org/10.1002/sim.2980.

Dow, W. H., Norton, E. C., \& Donahoe, J. T. (2019). Stata tip 134: Multiplicative and marginal interaction effects in nonlinear models. The Stata Journal, 19(4), 1015-1020. https://doi.org/10.1177/ $1536867 X 19893644$.

Dube, S. S. R. S., Felitti, V. V. J. V., Dong, M., Chapman, D. D. P. D., Giles, W. W. H., \& Anda, R. F. R. (2003). Childhood abuse, neglect, and household dysfunction and the risk of illicit drug use: The adverse childhood experiences study. Pediatrics, 111(3), 564-572. https://doi.org/10.1542/peds.111.3.564.

Duke, N. N., Pettingell, S. L., McMorris, B. J., \& Borowsky, I. W. (2010). Adolescent violence perpetration: associations with multiple types of adverse childhood experiences. Pediatrics, 125(4), e778-e786. https://doi.org/10.1542/peds.2009-0597.

Eiden, R. D., Godleski, S. A., Colder, C. R., Livingston, J. A., Leising, M. C., \& Leonard, K. E. (2020). Early childhood risk and protective factors predicting resilience against adolescent substance use. Adversity and Resilience Science, 1(2), 107-119. https://doi.org/ 10.1007/s42844-020-00007-5.

Elmore, A. L., \& Crouch, E. (2020). The Association of adverse childhood experiences with anxiety and depression for children and youth, 8 to 17 years of age. Academic Pediatrics, 20(5), 600-608. https://doi.org/10.1016/j.acap.2020.02.012.
Elmore, A. L., Crouch, E., \& Chowdhury, M. A. K. (2020). The Interaction of Adverse Childhood Experiences and Resiliency on the Outcome of Depression Among Children and Youth, 8-17 year olds. Child Abuse \& Neglect, 107, 104616. https://doi.org/10. 1016/j.chiabu.2020.104616.

Felitti, V. J., Anda, R. F., Nordenberg, D., Williamson, D. F., Spitz, A. M., Edwards, V., ... Marks, J. S. (2019). Relationship of childhood abuse and household dysfunction to many of the leading causes of death in adults: The adverse childhood experiences (ACE) study. American Journal of Preventive Medicine, 56(6), 774-786. https://doi.org/10.1016/j.amepre.2019.04.001.

Fergus, S., \& Zimmerman, M. A. (2005). Adolescent resilience: A framework for understanding healthy development in the face of risk. Annual Review of Public Health, 26, 399-419. https://doi. org/10.1146/annurev.publhealth.26.021304.144357.

Finkelhor, D. (2020). Trends in adverse childhood experiences (ACEs) in the United States. Child Abuse \& Neglect, 108, 104641. https:// doi.org/10.1016/j.chiabu.2020.104641.

Forster, M., Gower, A. L., Borowsky, I. W., \& McMorris, B. J. (2017). Associations between adverse childhood experiences, studentteacher relationships, and non-medical use of prescription medications among adolescents. Addictive Behaviors, 68, 30-34. https:// doi.org/10.1016/j.addbeh.2017.01.004.

Golberstein, E., Gonzales, G., \& Meara, E. (2019). How do economic downturns affect the mental health of children? Evidence from the National Health Interview Survey. Health Economics (united Kingdom), 28(8), 955-970. https://doi.org/10.1002/hec.3885.

Golberstein, E., Wen, H., \& Miller, B. F. (2020). Coronavirus disease 2019 (COVID-19) and mental health for children and adolescents. JAMA pediatrics, 174(9), 819-820. https://doi.org/10.1001/jamap ediatrics.2020.1456.

Hawkins, J. D., Oesterle, S., Brown, E. C., Monahan, K. C., Abbott, R. D., Arthur, M. W., \& Catalano, R. F. (2012). Sustained decreases in risk exposure and youth problem behaviors after installation of the communities that care prevention system in a randomized trial. Archives of Pediatrics and Adolescent Medicine, 166(2), 141-148. https://doi.org/10.1001/archpediatrics.2011.183.

Hays-Grudo, J., \& Morris, A. S. (2020). Adverse and protective childhood experiences: A developmental perspective. American Psychological Association. https://doi.org/10.1037/0000177-000.

Hays-Grudo, J., Morris, A. S., Beasley, L., Ciciolla, L., Shreffler, K., \& Croff, J. (2021). Integrating and synthesizing adversity and resilience knowledge and action: The ICARE model. The American Psychologist, 76(2), 203-215. https://doi.org/10.1037/amp00 00766.

Henry, C. S., Sheffield Morris, A., \& Harrist, A. W. (2015). Family Resilience: Moving into the Third Wave. Family Relations, 64(1), 22-43. https://doi.org/10.1111/fare.12106.

Hostinar, C. E., \& Miller, G. E. (2019). Protective factors for youth confronting economic hardship: Current challenges and future avenues in resilience research. American Psychologist, 74(6), 641-652. https://doi.org/10.1037/amp0000520.

Ivey-Stephenson, A. Z., Demissie, Z., Crosby, A. E., Stone, D. M., Gaylor, E., Wilkins, N., ... Brown, M. (2020). Suicidal Ideation and behaviors among high school students - Youth risk behavior survey, United States, 2019. MMWR Supplements, 69(1), 47-55. https://doi.org/10.15585/mmwr.su6901a6.

Javier, J. R., Hoffman, L. R., \& Shah, S. I. (2019). Making the case for ACEs: Adverse childhood experiences, obesity, and long-term health. Pediatric Research, 86(4), 420-422. https://doi.org/10. 1038/s41390-019-0509-2.

Keyes, K. M., Gary, D., O’Malley, P. M., Hamilton, A., \& Schulenberg, J. (2019). Recent increases in depressive symptoms among US adolescents: Trends from 1991 to 2018. Social Psychiatry and 
Psychiatric Epidemiology, 54(8), 987-996. https://doi.org/10. 1007/s00127-019-01697-8.

Keyes, K. M., Hamilton, A., Patrick, M. E., \& Schulenberg, J. (2020). Diverging Trends in the Relationship Between Binge Drinking and Depressive Symptoms Among Adolescents in the U.S. From 1991 Through 2018. Journal of Adolescent Health, 66(5), 529535. https://doi.org/10.1016/j.jadohealth.2019.08.026.

Kroenke, K., Spitzer, R. L., \& Williams, J. B. W. (2003). The patient health questionnaire-2: Validity of a two-item depression screener. Medical Care, 41(11), 1284-1292. https://doi.org/10.1097/01. MLR.0000093487.78664.3C.

Kroenke, K., Strine, T. W., Spitzer, R. L., Williams, J. B. W., Berry, J. T., \& Mokdad, A. H. (2009). The PHQ-8 as a measure of current depression in the general population. Journal of Affective Disorders, 114(1-3), 163-173. https://doi.org/10.1016/j.jad.2008. 06.026.

Leffert, N., Benson, P. L., Scales, P. C., Sharma, A. R., Drake, D. R., \& Blyth, D. A. (1998). Developmental assets: Measurement and prediction of risk behaviors among adolescents. Applied Developmental Science, 2(4), 209-230. https://doi.org/10.1207/s1532 480xads0204_4.

Liu, S. R., Kia-Keating, M., Nylund-Gibson, K., \& Barnett, M. L. (2020). Co-occurring youth profiles of adverse childhood experiences and protective factors: Associations with health, resilience, and racial disparities. American Journal of Community Psychology, 65(1-2), 173-186. https://doi.org/10.1002/ajcp.12387.

Lu, W. (2019). Adolescent depression: National trends, risk factors, and healthcare disparities. American Journal of Health Behavior, 43(1), 181-194. https://doi.org/10.5993/AJHB.43.1.15.

Luthar, S. S., Cicchetti, D., \& Becker, B. (2000). The construct of resilience: A critical evaluation and guidelines for future work. Child Development, 71(3), 543-562. https://doi.org/10.1111/ 1467-8624.00164.

Masten, A. S. (2001). Ordinary magic: Resilience processes in development. American Psychologist, 56(3), 227-238. https://doi.org/10. 1037/0003-066X.56.3.227.

Masten, A. S. (2015). Ordinary magic: Resilience in development. New York, NY: The Guilford Press.

Masten, A. S., \& Motti-Stefanidi, F. (2020). Multisystem resilience for children and youth in disaster: Reflections in the context of COVID-19. Adversity and Resilience Science, 1(2), 95-106. https://doi.org/10.1007/s42844-020-00010-w.

Masten, A. S., \& Reed, M. G. (2009). Resilience in development. In Handbook of positive psychology (pp.74-88). Oxford: Oxford University Press.

Minnesota Department of Health. (2019). Minnesota student survey reports 2013-2019. Retrieved from https://www.health.state.mn. us/news/pressrel/2019/studentsurvey101719.html.

Mojtabai, R., Olfson, M., \& Han, B. (2016). National trends in the prevalence and treatment of depression in adolescents and young adults. Pediatrics, 138(6), e20161878. https://doi.org/10.1542/ peds.2016-1878.

Morris, A. S., Hays-Grudo, J., Zapata, M. I., Treat, A., \& Kerr, K. L. (2021). Adverse and Protective Childhood Experiences and Parenting Attitudes: the Role of Cumulative Protection in Understanding Resilience. Adversity and Resilience Science, 1-12. https://doi.org/10.1007/s42844-021-00036-8.

Oesterle, S., Kuklinski, M. R., Hawkins, J. D., Skinner, M. L., Guttmannova, K., \& Rhew, I. C. (2018). Long-term effects of the communities that care trial on substance use, antisocial behavior, and violence through age 21 years. American Journal of Public Health, 108(5), 659-665. https://doi.org/10.2105/AJPH.2018. 304320.

Parks, M. J., Davis, L., Kingsbury, J. H., \& Shlafer, R. J. (2020). Adverse childhood experiences and youth cigarette use in 2013 and 2016: emerging disparities in the context of declining smoking rates. Nicotine \& Tobacco Research, 22(1), 124-129. https:// doi.org/10.1093/ntr/nty 178 .

Patrick, M. E., Couper, M. P., Parks, M. J., Laetz, V., \& Schulenberg, J. E. (2021). Comparison of a web-push survey research protocol with a mailed paper and pencil protocol in the Monitoring the Future panel survey. Addiction, 16(1), 191-199. https://doi.org/ 10.1111/add.15158.

Patton, G. C., Sawyer, S. M., Santelli, J. S., Ross, D. A., Afifi, R., Allen, N. B., ... \& Viner, R. M. (2016). Our future: a Lancet commission on adolescent health and wellbeing. The Lancet, 387(10036), 2423-2478. https://doi.org/10.1016/S0140-6736(16)00579-1.

Resnick, M. D., Bearman, P. S., Blum, R. W., Bauman, K. E., Harris, K. M., Jones, J., ... Udry, J. R. (1997). Protecting adolescent's from harm: Findings from the national longitudinal study on adolescent health. Journal of the American Medical Association, 278(10), 823-832. https://doi.org/10.1001/jama.278.10.823.

Richardson, L. P., McCauley, E., Grossman, D. C., McCarty, C. A., Richards, J., Russo, J. E., ... Katon, W. (2010). Evaluation of the patient health questionnaire-9 item for detecting major depression among adolescents. Pediatrics, 126(6), 1117-1123. https://doi. org/10.1542/peds.2010-0852.

Rutter, M. (1987). Psychosocial resilience and protective mechanisms. American Journal of Orthopsychiatry, 57(3), 316-331. https://doi. org/10.1111/j.1939-0025.1987.tb03541.x.

Sacks, V., \& Murphey, D. (2018). The prevalence of adverse childhood experiences, nationally, by state, and by race or ethnicity. Research brief. Child Trends, (February).

Sacks, V., Murphey, D., \& Moore, K. (2014). Research Brief: Adverse Childhood Experiences: National and State-Level Prevalence. Child Trends (Vol. 2011).

Sameroff, A. (1975). Transactional models in early social relations. Human Development, 18(1-2), 65-79. https://doi.org/10.1159/ 000271476.

Sameroff, A. J., Seifer, R., \& Bartko, W. T. (1997). Environmental perspectives on adaptation during childhood and adolescence. In Developmental Psychopathology: Perspectives on Adjustment, Risk, and Disorder (pp. 507-526). Cambridge: Cambridge University Press.

Scales, P. C. (1999). Reducing risks and building developmental assets: Essential actions for promoting adolescent health. Journal of School Health, 69(3), 113-119. https://doi.org/10.1111/j.17461561.1999.tb07219.x.

Scales, P. C., Benson, P. L., Leffert, N., \& Blyth, D. A. (2000). Contribution of developmental assets to the prediction of thriving among adolescents. Applied Developmental Science, 4(1), 27-46. https:// doi.org/10.1207/S1532480XADS0401_3.

Scales, P., Leffert, N., \& Lerner, R. (2004). Developmental assets: A synthesis of the scientific research on adolescent development. Search Institute.

Sesma, A., Mannes, M., \& Scales, P. C. (2013). "Positive adaptation, resilience and the developmental assets framework." In Handbook of Resilience in Children: Second Edition (pp. 427-442). New York, NY: Springer. https://doi.org/10.1007/978-1-4614-3661-4_ 25.

Spitzer, R. L., Kroenke, K., \& Williams, J. B. W. (1999). Validation and utility of a self-report version of PRIME-MD: The PHQ Primary Care Study. Journal of the American Medical Association, 282(18), 1737-1744. https://doi.org/10.1001/jama.282.18.1737.

Syvertsen, A. K., Scales, P. C., \& Toomey, R. B. (2021). Developmental assets framework revisited: Confirmatory analysis and invariance testing to create a new generation of assets measures for applied research. Applied Developmental Science, 25(4), 291-306. https://doi.org/10.1080/10888691.2019.1613155. 
Turney, K. (2020). Cumulative adverse childhood experiences and children's health. Children and Youth Services Review, 119, 105538. https://doi.org/10.1016/j.childyouth.2020.105538.

Weinberger, A. H., Gbedemah, M., Martinez, A. M., Nash, D., Galea, S., \& Goodwin, R. D. (2018). Trends in depression prevalence in the USA from 2005 to 2015: Widening disparities in vulnerable groups. Psychological Medicine, 48(8), 1308-1315. https://doi. org/10.1017/S0033291717002781.

Werner, E. E., \& Smith, R. S. (1992). Overcoming the odds: High risk children from birth to adulthood. Ithaca, NY: Cornell University Press.
Winslow, E. B., Sandler, I. N., Wolchik, S. A., \& Carr, C. (2013). "Building resilience in all children: A public health approach." In Handbook of Resilience in Children: Second Edition (pp. 459480). New York, NY: Springer. https://doi.org/10.1007/978-14614-3661-4_27.

Wright, M. O. D., Masten, A. S., \& Narayan, A. J. (2013). "Resilience processes in development: Four waves of research on positive adaptation in the context of adversity." In Handbook of Resilience in Children: Second Edition (pp. 15-37). New York, NY: Springer. https://doi.org/10.1007/978-1-4614-3661-4_2. 